\title{
Associations of sleep quality and night eating behaviour with gut microbiome composition in adults with metabolic syndrome
}

\author{
E. Bellikci-Koyu ${ }^{1,2}$, B.P. Sarer-Yurekli ${ }^{3}$, Y. Akyon ${ }^{4}$, A.G. Ozgen ${ }^{3}$, A. Brinkmann ${ }^{5}$, A. Nitsche ${ }^{5}$, \\ K. Ergunay ${ }^{4}$, E. Yilmaz ${ }^{6}$, M. P. St-Onge ${ }^{7}$ and Z. Buyuktuncer ${ }^{1}$ \\ ${ }^{1}$ Department of Nutrition and Dietetics, Faculty of Health Sciences, Hacettepe University, Turkey, \\ ${ }^{2}$ Department of Nutrition and Dietetics, Faculty of Health Sciences, Izmir Katip Celebi University, Turkey, \\ ${ }^{3}$ Department of Endocrinology, Faculty of Medicine, Ege University, Turkey, \\ ${ }^{4}$ Department of Medical Microbiology, Faculty of Medicine, Hacettepe University, Turkey, \\ ${ }^{5}$ Robert Koch Institute, Center for Biological Threats and Special Pathogens 1 (ZBS-1), Germany, \\ ${ }^{6}$ Department of Medical Biology, Faculty of Medicine, Hacettepe University, Turkey (Retired Professor) and \\ ${ }^{7}$ Sleep Center of Excellence \& Division of General Medicine, Department of Medicine, Columbia University Irving \\ Medical Center, New York, USA
}

Circadian misalignment induced by disruptions in sleep/wake cycle or feeding/fasting cycle has been linked with both metabolic health, including energy homeostasis and insulin sensitivity ${ }^{(1)}$ and alterations in gut microbiome ${ }^{(2)}$. This study aimed to examine the potential associations among sleep quality, night eating behaviour and gut microbiome composition in patients with metabolic syndrome.

This is a secondary analysis of data collected for a randomized controlled clinical trial ${ }^{(3)}$. The cross-sectional analysis was performed using the baseline data of twenty-one patients with metabolic syndrome (37-64 years; 16 females) who completed the Pittsburgh Sleep Quality Index Questionnaire (PSQI) for the evaluation of sleep quality and the Night Eating Questionnaire (NEQ) for the assessment of behavioural and psychological symptoms of night eating syndrome. Participants also provided stool samples for 16S ribosomal RNA gene sequencing analysis. Descriptive statistics, Spearman's correlation and partial correlation tests were computed using IBM SPSS Statistics 23 software. Partial correlation coefficients were calculated with adjustment for age, sex, body mass index and dietary fibre intake.

The median of PSQI score was 3.0 with a range of 1.0-17.0 and median score on NEQ was 16.0 (6.0-34.0). Sleep disturbances and night eating syndrome were observed in $4(19.0 \%)$ and $6(28.6 \%)$ participants, respectively. PSQI score was correlated with the NEQ score $(r=0.571, p=0.007)$, confirming a relationship between sleep quality and night eating behaviour. Sleep quality and night eating syndrome were associated with similar microbiome profiles. At the phylum level, poor sleep quality was correlated with higher relative abundance of Verrucomicrobia and Proteobacteria $(r=0.789$ and $r=0.833$ respectively, both $p<0.001)$, but lower relative abundance of Bacteroidetes $(\mathrm{r}=-0.569, \mathrm{p}=0.017)$. At the sub-phylum levels, poor sleep quality was correlated with Alistipes $(r=0.535, p=0.027)$ and Erysipelotrichaceae $(r=0.821, p<0.001)$ abundance. Similarly, the relative abundance of phyla Proteobacteria $(r=0.501, p=0.040)$, and genus Alistipes $(r=0.595, p=0.012)$ were associated with night eating behaviour. Partial correlations suggested that the relationships between bacterial abundance and night eating behaviour were not independent of sleep quality ( $\mathrm{r}=0.039, \mathrm{p}=0.886$ for Proteobacteria; $\mathrm{r}=0.413, \mathrm{p}=0.111$ for Alistipes).

This study suggests associations between gut microbiome composition and circadian disruptions induced by sleep disturbances or eating late at night. The associations between night eating behaviour and gut microbiome may be due in part to poor sleep quality. These data suggest that not only does eating at night, but also the sleep disruptions that result from this activity, may contribute to alterations in gut microbiome. Further research is required to identify the potential bidirectional relationship between circadian rhythms and gut microbiome that may affect overall health.

\section{References}

1. Morris CJ, Yang JN, Garcia JI, et al. (2015) Proc Natl Acad Sci USA 112(17), 2225-2234.

2. Qin J, Li Y, Cai Z, et al. (2012) Nature 490(7418), 55-60.

3. Bellikci-Koyu E, Yurekli BS, Akyon Y, et al. (2019) Nutrients 11, 2089. 